\title{
Adjusting insulin doses: from knowledge to decision
}

\author{
Gérard Reach $^{\mathrm{a}, *}$, Asmae Zerrouki ${ }^{\mathrm{b}}$, Dieudonné Leclercq ${ }^{\mathrm{c}}$, Jean-François d'Ivernois ${ }^{\mathrm{b}}$ \\ a INSERM U341, Service de Diabétologie, Hôtel-Dieu, Paris, and Service de Médecine Interne et Endocrinologie, Hôpital Avicenne, \\ Assistance Publique-Hôpitaux de Paris, 125 route de Stalingrad, 93000 Bobigny, France \\ ${ }^{\mathrm{b}}$ Laboratoire de Pédagogie de la Santé, UPRES EA 3412, Faculté de Médecine de Bobigny, Université Paris XIII, France \\ ' Service de Technologie de l'Education, Faculté de Psychologie et des Sciences de l'Education, Université de Liège, Belgium
}

Received 25 March 2002; received in revised form 8 July 2003; accepted 19 January 2004

\begin{abstract}
The aim of this study was to analyze the absence of adjustment of insulin doses in type 1 diabetic patients with poorly controlled diabetes. Twenty-eight patients $\left(\mathrm{HbA}_{1} \mathrm{c}\right.$ higher than $8.5 \%$ during the last 6 months, performing at least three capillary blood glucose determinations per day), completed a questionnaire on the degree of confidence in their own knowledge, the nature of their health beliefs, their fear of hypoglycemia, their own appreciation on how they adjust their insulin doses (subjective score). An analysis of their diabetes logbook provided an objective score of the adjustment of doses actually performed. The results show that the subjective and objective scores of adjustment were not significantly correlated. Further there was a significant negative correlation between the score of uncertainty on knowledge and the subjective score of adjustment of the insulin doses, but not with the objective score. There was a significant correlation between the score of positive health beliefs and the subjective score of adjustment of the insulin doses, but not with the objective score. No correlation was found between the score of fear of hypoglycemia and the subjective score of adjustment of the insulin doses. Correlation with the objective score was higher, but not significant. Actually, the fear of hypoglycemia was the most frequently given reason for not adjusting the insulin doses, when the question was asked to the patients with an open answer. This study illustrates the difference between thinking and doing. It also shows that the degree of confidence in one's own knowledge, the health beliefs, and the fear of hypoglycemia differently influence the perception that the patients have of their behavior, and what they really do.
\end{abstract}

(C) 2004 Elsevier Ireland Ltd. All rights reserved.

Keywords: Insulin therapy; Patient education; Causal theory of action; Health beliefs; Uncertainty on knowledge; Confidence degrees

\section{Introduction}

The objective of the health care provider in the treatment of type 1 diabetes is to obtain the best metabolic control in order to avoid long-term complications, while avoiding the occurrence of severe hypoglycemic episodes, and protecting the quality of life [1]. To these ends, patients are required to perform injections of insulin and measures of capillary blood glucose concentration several times a day. They learn how to realize these different tasks through educational programs provided by the health care team. Besides these tasks, they are taught appropriate responses to apply to some situations and how to put them into practice: for example, daily modification of the insulin doses to maintain glycaemia within limits defined by the medical team, or carrying sugar with them to correct for hypoglycemia [2].

The adjustment of the insulin doses requires complex reasoning taking into account the results of the insulin doses in-

\footnotetext{
* Corresponding author. Tel.: +33-1-48955158; fax: +33-1-48955560. E-mail address: gerard.reach@avc.ap-hop-paris.fr (G. Reach).
}

jected in the previous days, glycaemia at the time of injecting insulin, and the prediction of physical activity in the following period of the day. This complexity was highlighted both by the health care team and by the patients $[3,4]$. Clearly, reasoning is not enough. It must be followed by an actual application of the decision to change, or not, the insulin dosage. Unfortunately, a large number of patients never adjust their doses of insulin while their blood glucose levels are high, accounting in part for elevated glycated hemoglobin. This increases the risk for the development of long-term complications. This absence of adjustment of the insulin doses often occurs while other tasks such as injecting insulin, self blood glucose monitoring, and even the recording of the data in a diabetes logbook, are accomplished correctly $[5,6]$.

Several causes may underlie this behavior. A failure of the therapeutic education: patients do not adjust the insulin doses because they did not learn how to do it or because they did not understand the rules which were explained to them, or because they are not sure enough of their knowledge, uncertainty entailing indecision $[7,8]$. Further, in fact, it is clear that the therapeutic education of the patients is not restricted 
to a transmission of knowledge, but is also aimed to elicit behaviors from the patients [2]. The absence of adjustment of the insulin doses, in this frame, does not involve only cognitive factors, but may also be related to the following different reasons. A first concerns the patient's health beliefs [9], leading him/her, for example, to think that it is useless or impossible to try to normalize glycaemia, or the belief that insulin causes weight gain, or the belief that he/she does not know enough to be successful. A second one is due to the fear of hypoglycemia, so that the patient may prefer to leave this responsibility to the health care provider; indeed, severe hypoglycemia is the most feared complication of diabetes [10]. Another one is the lack of motivation, the patient refusing to spend time in the self-management tasks, or the simple fact that the repeated tasks bore the patient.

The aim of this study was therefore to examine the reasons for the absence of adjustment of insulin in type 1 diabetic patients with poorly controlled diabetes. This study used on one hand a questionnaire evaluating cognitive and behavioral items, and on the other hand examined the patient's own diabetes logbook to estimate the actual adjustment of the insulin doses.

\section{Methods}

Twenty-eight patients, followed by one of us (G.R.) for at least 5 years, who had a mean glycated hemoglobin level higher than $8.5 \%$ during the 6 months preceding the study, who measured their glycaemia at least three times a day, agreed to participate in this study. Table 1 represents the demographic of the patients and the main characteristics of their diabetes.

\subsection{Questionnaire}

The patients received a questionnaire during a medical visit, and it was clear that their answers would not be communicated to their diabetologist, in order not to bias their answers. They communicated their answers during a phone call (15-60 min) with one of us (A.Z). One of the purposes

Table 1

Characteristics of the patients

\begin{tabular}{|c|c|c|c|}
\hline & $N$ & Mean & Range \\
\hline Male/female & $11 / 17$ & & \\
\hline Family history of diabetes & & $29 \%$ & \\
\hline Presence of retinopathy & & $36 \%$ & \\
\hline Presence of a nephropathy & & $4 \%$ & \\
\hline Presence of hypertension & & $20 \%$ & \\
\hline Age (years) & & $33 \pm 14$ & $18-68$ \\
\hline Diabetes duration & & $16 \pm 9$ & $6-40$ \\
\hline $\mathrm{HbA}_{1} \mathrm{c}(\%)$ & & $9.4 \pm 0.9$ & $8.2-12.1$ \\
\hline Injections of insulin per day & & $3.8 \pm 0.6$ & $2-5$ \\
\hline Blood glucose determinations per day & & $3.8 \pm 0.9$ & $3-6$ \\
\hline Visits with the diabetologist per year & & $5.5 \pm 3.0$ & $2-15$ \\
\hline
\end{tabular}

of the call was to make sure that the questionnaire had been fully completed. In addition, an objective analysis of the adjustments of the insulin doses was done (see below) on photocopies of the pages of the logbook covering the three previous months.

The questionnaire contained, besides the demographic questions (Section 1, Table 1), 61 true/false questions and one open-ended question, put at the end of questionnaire ("why do you avoid to increase your insulin dose while your blood glucose levels are high?"). Closed questions were organized in several sections: the Section 2 (30 questions) aimed to determine the level of knowledge of the patients by proposing a sentence of the type "hypoglycemia can be caused by too much insulin. True-false", or "If, when you wake up, glycaemia is lower than $5.5 \mathrm{mmol} / \mathrm{l}$, you should decrease the dose of insulin injected in the evening. True-false". Subjects were asked to add a confidence degree to their answer $[7,8]$, indicating whether they were sure of it at 2\% (not at all sure), $10 \%$ (not sure), 25\% (weakly sure), $50 \%$ (does not know), $75 \%$ (rather sure), $90 \%$ (sure), $98 \%$ (completely sure). There were 14 propositions concerning theoretical aspects, 3 questions on practical tasks and 13 questions concerning practical knowledge, given in a random order. Section 3 of the questionnaire (11 questions) concerned the health beliefs, for example "You can act effectively to improve your health" with a gradation of possible answers according to a Lickert's scale (total or partial disagreement, partial or total agreement) [11]. Section 4 (10 questions) concerned hypoglycemia, for example "Do you carry some sugar with you?", "Did you experience a hypoglycemia episode for which the intervention of another person was necessary?", the answers being also organized according to a Lickert's scale in four levels. Section 5 (10 questions) grouped questions concerning time devoted to the self-management (five questions), and the possible existence of smoking (two questions). Finally, the last three questions indicated to the patients "that their aim is to clarify whether you really practice the adjustment of the insulin doses: they do not test your knowledge, but what you do really". For example: "In case of hypoglycemia, do you really decrease the dose of insulin which covers the period when it occurred?" (possible answers: never, rarely, mostly, always).

From the answers to these questions, the following scores were established: (i) subjective score of the adjustment of the insulin doses; (ii) score of positive health beliefs; (iii) score of knowledge uncertainty, established on the whole set of knowledge questions, and by distinguishing knowledge related to theoretical or practical notions; according to $[7,8,12-14]$, this score was established by giving for each question a score of 0 (when confidence is sure at 98 or $90 \%$ ) and 1 (tendency to uncertainty) if the answer was equal or lower than $75 \%$ of certitude; (iv) score of clarity of explanations provided by the health care providers; (v) score of constraint due to the time devoted to treatment, (vi) score of fear of hypoglycemia. These scores were obtained by adding the values given on the respective Lickert's scales. 
Concerning the open question, we used the content analysis methodology described by Gravitz [15].

The second part of the study consisted in an objective analysis, of the pages of the logbook for the previous 3 months, and in establishing an objective score of adjustment of the insulin doses actually practiced by the patients, as $I=b / a+d / c$ (possible value between 0 and 2), where (a) is the number of capillary blood glucose determinations higher than $10 \mathrm{mmol} / \mathrm{l}$ at the time of rapid insulin injection, not preceded by a hypoglycemia episode, (b) is the number of cases where these high blood glucose levels were followed by an increase in the dose of rapid acting insulin; (c) is the number of weeks where the average glycaemias at wake up was higher than $10 \mathrm{mmol} / \mathrm{l}$ without nocturnal hypoglycemia; (d) is the number of weeks where these high average glycaemias entailed an increase in the dose of intermediate or slow action insulin injected in the evening.

\subsection{Data analysis}

All results in text and figures are given as mean \pm S.E.M. In the analysis e correlation coefficient of Spearman are used. For 28 subjects, a level of statistical significance of $P<0.05$ is obtained for $r>0.317$, and $P<0.01$ is obtained for $r>0.432$.

\section{Results}

\subsection{Adjustment of the insulin doses: subjective and objective scores}

The subjective score of adjustment of the insulin doses was obtained by asking the patients whether they thought that they adjust their insulin doses, while the objective score was calculated by analyzing the logbook of treatment. They were $21 \pm 3$ (range 14-27, median 21, the highest possible score being 31 ) and $0.68 \pm 0.52$ (range 0 to 1.84 , median 0.65 , a perfect adjustment would have given a score of 2), respectively. The two scores were not significantly correlated $(r=0.254$, NS) (see Fig. 1). The dispersion of points on this figure shows that there were nine patients out of 28 who either claimed that they adjusted the insulin doses (subjective score superior to the median 21) while actually they did not do it (objective score lower than the median 0.65), or that they thought that they did not adjust their insulin doses, while they actually did it.

\subsection{Cognitive aspects of the adjustment of the insulin doses}

The percentage of questions for which the patients answered that they were not sure of their answer (below or equal to $75 \%$ of certitude) was $36 \pm 27 \%$ (range $0-93 \%$ ). There was a strong correlation between the score of uncertainty expressed as the percentage of questions for which the patients declared to be uncertain, concerning practical and theoretical knowledge $(y=1.05 x+7.96, r=0.892)$, the slope close to 1 suggesting that the degree of uncertainty on the two types of knowledge was identical.

The degree of uncertainty was negatively correlated to the clarity of explanations, the correlation being statistically significant $(P<0.05)$ for the whole set of questions $(r=$ $0.430)$ and practical questions $(r=0.465)$, and close to the statistical significance $(r=0.315)$ for the theoretical questions.

\subsection{Uncertainty on knowledge and subjective score of adjustment of the insulin doses}

There was a significant negative correlation $(r=0.41$, $P<0.05)$ between the subjective score of insulin and the

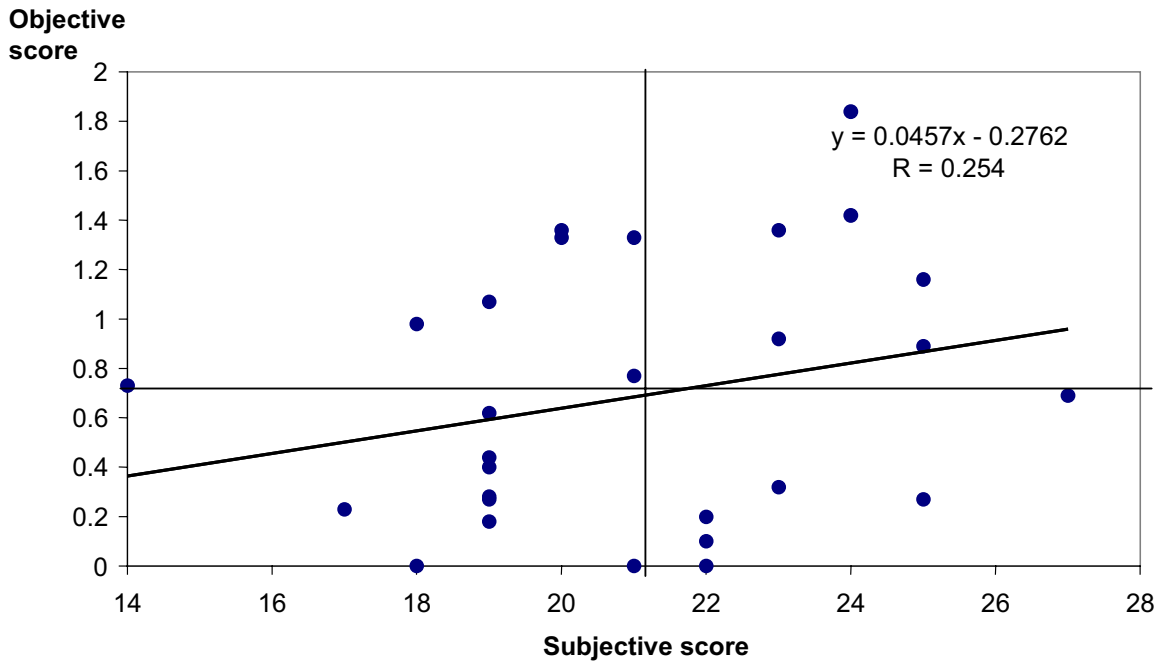

Fig. 1. Correlation between the objective and subjective scores of adjustment of the insulin doses (NS). The horizontal and vertical lines give the median values of scores. 
score of uncertainty on knowledge, calculated on the whole set of tested knowledge. By contrast, this correlation was not found with the objective score of adjustment of the insulin doses $(r=0.27, \mathrm{NS})$.

\subsection{Health beliefs, adjustment of the insulin doses, time considered as a constraint, and degree of uncertainties on knowledge}

The mean score of positive health beliefs was $21.9 \pm 2.7$ (the highest possible score being 28). There was a significant correlation $(r=0.387, P<0.05)$ between the subjective score of adjustment of insulin and the score of positive health beliefs. On the other hand, no correlation was found between the objective score of adjustment of insulin and the score of positive health beliefs $(r=0.223$, NS). A significant negative correlation $(r=-0.385, P<0.05)$ was also found between a score indicating that the patient considers time devoted to the treatment as a constraint and the score of positive health beliefs. On the other hand, no correlation was found between the score of positive health beliefs and the score of uncertainty concerning the whole set of tested knowledge ( $r=0.05$, NS).

\subsection{Fear of hypoglycemia and absence of adjustment of the insulin doses}

No correlation was found between the subjective score of adjustment of insulin and the score of fear of hypoglycemia $(r=0.05, \mathrm{NS})$. Correlation with the objective score was higher, but not reaching statistical significance $(r=0.24$, NS).

\subsection{Carrying sugar to treat hypoglycemia}

Carrying sugar correlated significantly $(r=0.336)$ with the score of positive health beliefs, but neither with the fact of having experienced mild ( $r=0.13$, NS), or severe $(r=$ 0.08 , NS) episodes of hypoglycemia, nor with the subjective $(r=0.01, \mathrm{NS})$, or objective $(r=0.10$, NS), scores of

Table 2

Categorization of the answers to the question asked in an opened way: why do you avoid you to increase your dose of insulin while your blood glucose levels are high (some of the patients gave more than one answer)

\begin{tabular}{lcr}
\hline Given motive & Number of patient & $\%$ \\
\hline Fear of hypoglycemia & 12 & 37.5 \\
Gain weight & 6 & 18.8 \\
Difficult management of activities & 3 & 9.4 \\
Incomprehension & 3 & 9.4 \\
Negligence & 3 & 9.4 \\
Exceptional events & 2 & 6.3 \\
Family (fear of hypoglycemia) & 1 & 3.1 \\
It is a medical decision & 1 & 3.1 \\
Denial of the disease & 1 & 3.1 \\
Total & 32 & 100 \\
\hline
\end{tabular}

adjustment of the insulin doses. Correlation with the fear of hypoglycemia, although higher, did not reach the statistical significance $(r=0.24$, NS).

\subsection{Result of the question with opened answers}

Table 2 represents the motivations given by the patients when one asked them to clarify the reason for which they did not increase their insulin doses while their blood glucose determinations are high. The fears of hypoglycemia and of gain weight appear as the most frequent reasons.

\section{Discussion and conclusion}

We recently suggested [5] to consider any therapeutic task as a voluntary action which can be analyzed according to the causal theory of action developed by Davidson [15]. According to this theory, one does something when one has a reason to do it, and this reason is a pro-attitude, made of a desire (for example here the desire to avoid in the long term the complications of diabetes) and of a belief (for example, the belief that the fact of increasing the dose of insulin when glycaemia is high goes in this direction). Thus the fact of not adjusting the insulin doses (an example of therapeutic non-observance) can also be considered as a voluntary action, caused by the conjunction of a desire-for example to avoid hypoglycemia, to avoid weight gain - and the belief that the fact of not increasing the dose of insulin, even when glycaemia is high, belongs to the actions which will accomplish this desire [5]. Together with desires and beliefs other mental states intervene, described as propositional attitudes, such as emotions (fears, regrets, shames, etc. ... ) which, as desires and beliefs, have a content. These attitudes can lead to an assertion (the statement of the contents of the attitude) or, concerning beliefs, to an assent (the aware and voluntary acceptance of the content of the belief), and finally to an action [16]. As discussed by Engel [16], the assertion of the content of a belief is neither a necessary condition for the existence of this belief (one can believe a matter without ever expressing it), nor a sufficient condition (one can express a proposition without believing in it). Thus, if a subject acts according to a certain belief, without expressing it, this means that he nevertheless gave his assent to the content of this belief. On the other hand, the assent, as the assertion, is not a condition sufficient for the action, (one can approve the content of a belief without ever putting it into practice), nor necessary (one can act without approving the content of the belief which is behind this action).

\subsection{Discussion}

According to this novel conceptual framework, we will consider that the fact of actually adjusting the insulin doses or not, or of carrying pieces of sugar or not, represent two types of actions. By contrast, phenomenologically, thinking 
that one adjusts the insulin doses, having with more or less certitudes some knowledge, having positive health beliefs or not, being afraid of hypoglycemia, represent propositional attitudes.

Thus, in order to know the content of one of these attitudes, for instance whether a patient believes that it is good to adjust the insulin doses according to the results of the measures of the glycaemia, the best is not to ask him whether he/she believes it, or whether he/she believes that he/she puts this idea into practice, but to look in his/her logbook of treatment whether he/she did it actually. Indeed, as illustrated by this study the fact that one believes, and that one expresses the content of the belief, that one adjusts the insulin doses (subjective score), does not imply that one does it really (objective score) and inversely. Indeed, the first result of this study was the absence of statistically significant correlation between the subjective and objective scores of adjustment of the insulin doses. This absence of correlation can be due to the small number of subjects because there seems to be a trend (see Fig. 1), or to the fact that the methods of evaluation of two scores are not precise enough. However, it may also suggest that there is really a difference between thinking that one adjusts the insulin doses and actually doing it.

The second result of this study concerned our patients' knowledge uncertainty. For these 28 patients, the percentage of questions for which they were not sure of their answer varied from 0 to $93 \%$. However, there was a strong correlation between the percentages concerning theoretical questions and practical questions, although they were asked in a random order: in other words, some patients doubt about everything, and others do not doubt about anything. As expected, the degree of uncertainty was significantly correlated in a negative way to the representation by the patients of the clarity of the explanations which had been given to them.

According to the general conceptual framework used herein, knowledge uncertainty may be considered as an indicator of the strength of a propositional attitude. We observed that it can be correlated with the subjective feeling which the patients have to adjust the insulin doses, which is another propositional attitude, but not necessarily translated into an action, the fact of adjusting really the insulin doses, estimated by the objective score. Indeed, the more the patients were uncertain in their knowledge, the lower was their subjective score of adjustment of their insulin doses. This correlation was however not significant when the objective score of adjustment of the insulin doses was concerned, suggesting that uncertainty on knowledge had an influence on the representation that the patients have of their capacity to decide the dose of insulin, but not on their ability to do it actually. The absence of correlation between knowledge uncertainty and the objective score of adjustment of the insulin doses could suggest that the patients, when they adjust their insulin doses, do not use in fact "knowledge" which was taught to them to adjust the insulin doses, but rules which they learnt by themselves. There may be here an analogy with other problem solving situations, such as car driving, or chess playing [17].

The same effect can be observed for another propositional attitude, the positive health beliefs: as expected, they were found significantly correlated to the subjective score of adjustment of the insulin doses, but not to the objective score. Consistent with what one may expect intuitively, the score of positive health beliefs was significantly correlated in a negative way to the score estimating the constraint represented by the time devoted to the treatment, and in a positive manner to the fact of carrying sugar to treat hypoglycemia, this last item being not explained by past experiences of severe hypoglycemic episodes (maybe because the fact of carrying sugar prevented the occurrence of such accidents).

By contrast, there was no correlation between the fact of carrying sugar and the subjective score of adjustment of the insulin doses or the objective score. Similarly, a total lack of correlation was found between the score of positive health beliefs and the score of knowledge uncertainty, suggesting that mental states examined in these analyses are completely independent.

The last, but not least interesting, results concerned the fear of hypoglycemia. While, in an opened question, the fear of hypoglycemia emerged as the first cause expressed by the patients to explain the absence of adjustment of the insulin doses, the analysis of the questionnaire with true/false answers did not show any correlation between the score of fear of hypoglycemia, neither with the subjective score of adjustment of the insulin doses, nor with the objective score. This may represent an example where a reason, here an emotion, given by an agent to explain a behavior, may not have actually a strong effect, neither on the representation of his/her action, nor on what he/she actually does.

\subsection{Practice implications}

Educational diagnosis, the first step of any educational process [18], is aimed to explore, for a given topic, the content of these different "mental states", or propositional attitudes, of the patient. In conclusion, with all the limits of a study which concerns only 28 subjects treated by the same physician, data presented herein suggest that it may not only be necessary, in the framework of this educational diagnosis, to know about a given subject what the patients believe, wish, are afraid of, regret, etc., but also to evaluate the reality of their actions. Indeed, as shown in this study, there is a difference between what the patients claim, or even think, that they do, and what they actually do, as well as that there is a difference between the expressed, and the real, motivations of their actions.

\section{References}

[1] Assal JP. Traitement des maladies de longue durée: de la phase aiguë au stade de la chronicité. Une autre gestion de la maladie, un 
autre processus de la prise en charge (The treatment of long-term illness: transition from the acute to the chronic state. A different management of illness, a new approach). Encycl Méd Chir 1996;25-005-A-10:1-18.

[2] Bonnet C, Gagnayre R, d'Ivernois JF. Learning difficulties of diabetic educators. Patient Educ Couns 1998;35:139-47.

[3] Bonnet C, Gagnayre R, d'Ivernois JF. Difficulties of diabetic patients in learning about their illness. Patient Educ Couns 2001;42:159-64.

[4] Lombrail P, Obadia G, Thibult N, Eschwège E, Passa Ph. Absence de bénéfice de l'autosurveillance glycémique chez des diabétiques insulino-traités suivis en routine dans un service spécialisé en diabétologie (Lack of benefit of blood glucose autosurveillance in insulin-treated diabetics routinely followed up in a department specializing in diabetology). Presse Med 1986;15:1909-12.

[5] Reach G. Application de la théorie causale de l'action à l'analyse de la non-observance thérapeutique (Application of the causal theory of action through analysis of therapeutic non-observance). Presse Med 2000;29:1939-46.

[6] Leclercq D. Confidence marking, its use in testing. In: Choppin, Postlethwaite, editors. Evaluation in education: an international review Series. Pergamon Press; 1982. p. 161-287.

[7] Leclercq D. Approche technologique de l'éducation et de la formation (technological approach of education and training). Liège: Université de Liège, Faculté de Psychologie et des Sciences de l'Education; 1996.

[8] Becker M, Janz N. The health belief model applied to understanding diabetes regimen compliance. Diabetes Educ 1985;11:41-7.
[9] McCrimmon RJ, Frier BM. Hypoglycaemia, the most feared complication of insulin therapy. Diabete Metab 1994;20:503-12.

[10] Ebel RL. Essentials of educational measurement. Englewood Cliffs, NJ: Prentice-Hall Education Series; 1972.

[11] Leclerc D. Validity, reliability and acuity of self-assessment in educational testing. In: Leclercq D, Bruno J, editors. Item banking, interactive testing and self-assessment, NTO ARWASI Series F112. Heidelberg: Springer Verlag; 1993. p. 114-31.

[12] Jans V, Leclercq D. Metacognitive realism: a cognitive style or a learning strategy?, vol. 17, nos. 1 and 2. Educational psychology, special issue of learning and strategies; 1997. p. 101-10.

[13] Bruttomesso D, Gagnayre R, Leclercq D, Crazzolara D, Busata E, d'Ivernois JF, Casiglia E, Tiengo A, Baritussio A. The use of degrees of certainty to evaluate knowledge. Patient Educ Couns, 2004 [in press].

[14] Grawitz M. Méthodes des sciences sociales (Methods in social sciences), $10^{\text {ème }}$ ed. Daloz, Paris; 1996.

[15] Davidson D. Actions, reasons and causes. In: Davidson D, editor. Essays on actions and events. Oxford: Clarendon Press; 1980. p. 3-19.

[16] Engel P. Les croyances (Beliefs). In: Kambouchner D, editor. Notions de philosophie. Paris: Gallimard; 1995. p. 1-101.

[17] Dreyfus HL. La portée philosphique du connexionnisme (The philosophical implication of connexionism), In: Andler D, editor. Introductions aux sciences cognitives. Paris: Gallimard; 1992. p. 35273.

[18] d'Ivernois JF, Gagnayre R. Apprendre à éduquer le patient (Learning to educate the patient: pedagogical approach). Paris: Vigot; 1995. 